\title{
Zum Diskurs über die Sprache in der Wissenschaftskommunikation
}

\author{
Der Diskurs über die Wissenschaftssprache darf nicht von außerwissenschaft- \\ lichen Interessen bestimmt werden. Er muss in den Bereich der Wissenschaft \\ zurückgeholt und dort freiheitlich mit wissenschaftlicher Rationalität geführt
} werden. | Von Prof. Dr. Olga Rösch und Prof. Dr. Günter-Ulrich Tolkiehn

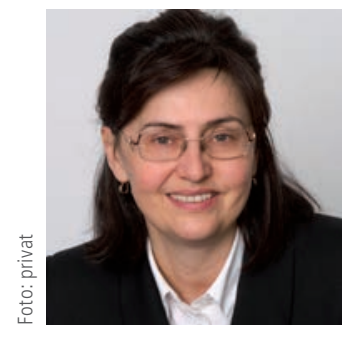

Prof. Dr. phil. Olga Rösch Professur für Interkulturelle Kommunikation

Technische Hochschule Wildau FB Ingenieur- und Naturwissenschaften Hochschulring 1 15745 Wildau

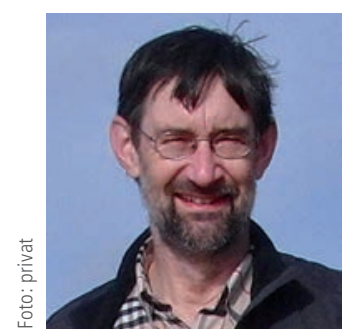

Prof. i. R. Dr. rer. nat. Günter-Ulrich Tolkiehn Wirtschaftsinformatik

TH Wildau

tolkiehn@th-wildau.de
Eines der beachtenswerten Kennzeichen der neuzeitlichen europäischen Landesbzw. Nationalsprachen ist die Existenz von ausgebildeten Wissenschaftssprachen. Damit sind nicht allein die wissenschaftlichen Fachsprachen gemeint, die gleichwohl ein Teil des Begriffssystems bzw. der begrifflichen Systematik von Wissenschaftssprachen sind, sondern die „Gesamtheit der Phänomene sprachlicher Tätigkeit (...), die im kulturellen Handlungsfeld der Wissenschaften auftreten und die zugleich dieses als theoriebildende und verarbeitende Kommunikationsgemeinschaft sowie als gesellschaftliche Institution entscheidend konstituieren" 1 . In ihrer Ausprägung als „wissenschaftliche Alltagssprache“ ist sie jenseits von Fachsprachen „die alles verbindende wissenschaftliche Sprache des Alltags von Forschung und Lehre" 2 , die sowohl mündliche als auch schriftliche Wissenschaftskommunikation einbegreift. Die historisch gewachsenen europäischen Wissenschaftssprachen sind Kulturgut und somit ein Teil der jeweiligen sprachlichen bzw. nationalkulturellen Identität - so wie die Mehrsprachigkeit des europäischen Kontinents ein konstituierender Bestandteil der europäischen Identität ist.

Gleichwohl besteht ein anhaltender Trend zur Verwendung des Englischen in Forschung und neuerdings auch Lehre. Er führt zur Verdrängung der nicht anglophonen Landessprachen aus der nationalen Wissenschaftskommunikation und wird inzwischen von vielen europäischen Wissenschaftlern kritisch gesehen. ${ }^{3}$ Der Widerspruch äußert sich in Appellen an die wissenschaftliche Öffentlichkeit ${ }^{4}$ sowie in Forderungen an die Nationalregierungen nach politischen und juristischen Maßnahmen zum Schutz der jeweiligen Wissenschaftssprachen. Im Ergebnis dieser Gegenbewegung wurden z. B. in Schweden 2009 und in Frankreich 2013 die sog. „Sprachgesetze“ verabschiedet, die die Landessprachen als Bildungssprachen stärken sollen. In Italien wurde 2018 per Gerichtsurteil erreicht, dass komplett englischsprachige Studiengänge nur dann zulässig sind, wenn es sie parallel auch auf Italienisch gibt. 5

Diese gegenläufigen Tendenzen - die voranschreitende Monolingualisierung der europäischen Wissenschaftskommunikation auf der einen Seite und die einsetzende Reflexion über die negativen Auswirkungen auf der anderen - ließen vielerorts in Europa einen „Sprach“-Diskurs entstehen. Es geht dabei im Grunde um ein bildungs- und wissenschaftspolitisches, kulturelles, ja sogar gesamtgesellschaftliches Anliegen.

Im neoliberalistisch dominierten Diskurs in Deutschland wird die Anglophonisierung der nationalen Wissenschaftskommunikation gern als „Modernität" proklamiert und Kritiker als „nicht auf der Höhe der Zeit" 6 diskreditiert. Auffallend ist indessen, dass der Begriff „Bildung“ in diesem Zusammenhang immer weniger erwähnt wird. Vordergründig wird pragmatisch mit Nützlichkeit ("praktisch“, „anschlussfähig“) argumentiert, ökonomisch („notwendig“) mit dem alles rechtfertigenden Begriff Globalisierung unterlegt und ideologisch mit positiv besetzten Begriffen („weltoffen“, „international“) konnotiert. Strukturell findet die Transformation in die Fremdsprache durch diverse DAAD-Programme, DFG-Förderungen $u$. a. (also institutionell und mit Steuergeld) breite sichere Stütze. Auch der Freiheit einer jeden Hochschulleitung, die 
Landessprache der Lehre per Verwaltungsakt durch das Englische zu ersetzen 7 oder Professoren durch „Leistungszulagen“ zur Lehre in englischer Sprache zu motivieren ${ }^{8}$, steht scheinbar fast nichts im Wege.

Der kritischen Analyse im deutschsprachigen Raum widmen sich zahlreiche Publikationen ${ }^{9}$ und es werden viele diesbezügliche Fachtagungen durchgeführt ${ }^{10}$. Eine von ihnen fand unter dem Titel „Die Sprache von Forschung und Lehre. Lenkung durch die Konzepte der Ökonomie?" im Februar 2018 in Tutzing statt.

\section{Die wesentlichen Diskussionsschwerpunkte der Tutzinger Tagung}

Die Konferenz war prominent besetzt, gut besucht und medial angemessen abgedeckt. ${ }^{11}$ Die Teilnehmer kamen aus vielen Fachrichtungen und aus verschiedenen europäischen Ländern. Der rote Faden, der sich durch die Vorträge und Diskussionen zog, lässt sich wie folgt zusammenfassen: Die Monolingualisierung der Wissenschaftskommunikation in Forschung und Lehre geht mittlerweile erheblich über die weithin anerkannte Verwendung von Englisch als kleinstem gemeinsamen Nenner für die globale wissenschaftliche Kommunikation per Konferenz und Publikation hinaus. Die Internationalität der Wissenschaft, zu deren Wesen die Vielfalt der Wissenschaftssprachen, Wissenschaftstraditionen und Kulturen gehört, wird so untergraben; die umfassende Einführung einer Einheitssprache in Forschung und Lehre in Europa würde den Charakter unseres Kontinents verleugnen; diesen Vorgang der Errichtung einer Sprachhegemonie Internationalisierung zu nennen, ist manipulativ; man kann ihn als eine Form der kulturellen Kolonialisierung mittels Sprache (Sprachfrage als Machtfrage) sehen und damit als Gegenentwurf zu gleichberechtigter Internationalität und Interkulturalität, die von Kontakt, Austausch und kultureller Bereicherung leben. Entsprechend der Fragestellung der Konferenz wurden die gegenwärtigen Entwicklungen in Forschung und Lehre kritisch reflektiert.

\subsection{Forschung}

Den Schwerpunkt im Bereich Forschung bildete die Publikationspraxis und ihre Verkennzahlung. Bei den wissenschaftlichen Verlagen entwickelte sich eine Zusammenarbeit mit rein numerisch arbeitenden Bewertungssystemen (i10-Index, h-Index, impact-factor, Zitationszahlen etc.). Die Übernahme dieser wissenschaftsexternen Bewertungen (zählen statt lesen) anstelle fachlicher Einschätzungen auch in Personal- und Drittmittelentscheidungen beklagen inzwischen auch die Wissenschaftler der anglophonen Länder. Für alle nicht anglophonen Länder kommt das Problem der sprachlichen Diskriminierung durch die bibliometrischen Indizes erschwerend hinzu. Dies verursacht einen enormen Druck in Richtung Monolingualisierung, der weit über den inhaltlichen Verständigungsbedarf hinausgeht und durch Definitions- und Deutungshoheit auch die Theoriebildung maßgeblich beeinflusst. Die Entfremdung zwischen der Öffentlichkeit und ihrer Wissenschaft wird so durch eine Sprach- und Kostenbarriere zusätzlich verstärkt.

Als Forderungen an die Politik, wie man dem negativen Trend im Forschungsbereich entgegenwirken kann, wurde erneut die Entwicklung von Anreizsystemen für Publikationen in der Muttersprache (ggf. zusätzlich zu englischsprachigen Veröffentlichungen) genannt ${ }^{12}$, um z. B. mithilfe eigener bibliometrischer Instrumente auf der EU-Ebene ein sprachdiskriminierungsfreies Regulativ zu schaffen. Die europäische Mehrsprachigkeit soll immer wieder ins Bewusstsein gerufen werden, denn die Vielsprachigkeit ist das Grundkonzept der EU. Die Wissensvermittlung des eigenen Faches in Landessprache(n) in die Gesellschaft soll gefördert werden, um die Verständlichkeit, Akzeptanz und Verwertung der Wissenschaft zu steigern. Auch soll der Austausch der Wissenschaft mit Politik und Wirtschaft intensiviert werden.

\subsection{Lehre}

Das Thema Englisch in der Lehre wurde besonders leidenschaftlich diskutiert. Dabei wurde immer wieder an den Bildungsauftrag der Hochschule erinnert: Der Gewinn der Bildung geht verloren, wenn die Studierenden keine originären akademischen Sprachen und Kulturen des eigenen Landes kennenlernen. Dies führt zur "Sprachlosigkeit“ in der Muttersprache und kommt einer kulturellen Entwurzelung gleich. Ernsthafte wissenschaftliche Lehre ist in einer Lingua Franca (in der Realität oft „bad English“) zwangsläufig zweitklassig und das umso stärker, je mehr das Fach auf Sprache angewiesen ist. Die in der Landessprache verfügbaren Fachtexte und Lehrinhalte werden entwertet und die Kommunikation zwischen Wissenschaft und anderen gesellschaftlichen Systemen, insb. der Politik und der Wirtschaft („Technologietransfer“) und der Gesellschaft insgesamt („Third Mission“), wird behindert. Englisch ist auch nicht „neutral“ wie etwa Esperanto. Anglophonisierung fördert eine unreflektierte Nachahmung von Denkstrukturen und Kulturelementen bzw. dessen, was man (aus der Provinzperspektive) dafür hält.

Für Erwerbstätigkeit in einem internationalen Unternehmen kann ein englischsprachiges Studium vorteilhaft sein. Das betrifft aber nur eine Randgruppe. Die breite Masse der Jobs erfordert Landessprache. Die Mehrzahl der ausländischen Studierenden, die übrigens mehrheitlich Englisch allenfalls als Zweitsprache sprechen, möchte später in Deutschland bleiben. ${ }^{13}$ Englischsprachige Studiengänge stellen für sie mehrheitlich ein Integrationshemmnis („Nowhere-Island“) ${ }^{14}$ und einen Nachteil auf dem deutschen Arbeitsmarkt dar. Deshalb soll der Anteil des deutschsprachigen Lehrangebots auch in grundsätzlich englischsprachigen Studiengängen im Laufe des Studiums immer mehr erhöht werden. Der zu 
beobachtende Abbau des professionellen Fremdsprachenunterrichts an den Hochschulen soll gestoppt werden, denn Internationalisierung erfordert akademische Mehrsprachigkeit.

\subsection{Einige Schlussbemerkungen zu der Tagung}

Die Positionen zur Stärkung der Landessprache und der Mehrsprachigkeit in der Lehre wurden in den „Tutzinger Forderungen zu Sprache der Lehre an deutschen Hochschulen “ 15 festgehalten. Interessant war zum Ende der Tagung die Einsicht, dass abgesehen von den politischen Interessen der USA und den wirtschaftlichen Interessen der beteiligten Unternehmen die „externen Treiber“ für die Anglisierung nicht überschätzt werden sollten. Was speziell Deutschland betrifft, sind es zum Teil auch die Deutschen selbst, die ungeprüft glauben, dass der Bedarf an Englisch so groß ist, die sich nur fortschrittlich, modern und international fühlen, wenn sie Englisch sprechen, oder Angst vor einer „Verdachtshermeneutik“ haben, die hinter jeder Bemühung um die deutsche Sprache nationalistisches Gedankengut vermutet. Diese selbst verordnete Anglophonisierung muss hinterfragt werden. Außerdem wurde festgehalten, dass es längst überfällig ist, sich vom Paradigma „Internationalisierung ist gleich Englisch“ zu verabschieden. ${ }^{16}$ Auffallend war bei der Diskussion, dass die Befürworter der Lehre in Englisch eine Sachargumentation, die über das Übliche („praktisch“, „weltoffen“, „Globalisierung“ u. Ä.) hinausging, nicht für nötig hielten.

\section{Zum ökonomischen Argument}

Eines der vielfach reproduzierten Internationalisierungs-Narrative, das in der Tagung nur am Rande kritisch diskutiert wurde, lautet: Deutschland befindet sich im internationalen Wettbewerb um „die besten Köpfe“ für den ständig von „Fachkräftemangel“ bedrohten deutschen Arbeitsmarkt und werde umso wettbewerbsfähiger, je mehr ausländische Studierende es durch englischsprachige Studienangebote gewinnen könne. Allerdings kommen ausländische Studierwillige in Deutschland mehrheitlich aus nicht anglophonen Ländern. Der Punkt „Studiengang wird in Englisch angeboten" fand sich mit 12 Prozent an der vorletzten Stelle von 13 abgefragten Gründen für die Wahl eines Studiums in Deutschland. ${ }^{17}$

Während die Vollfinanzierung der Hochschulausbildung inländischer Studierender für den Steuerzahler eine lohnende Investition ist 18 , ist das für ausländische Studierende ohne Kenntnisse der Landessprache durchaus nicht so klar. Die volkswirtschaftliche Rendite hängt hier entscheidend vom Prozentsatz der später im Studienland Erwerbstätigen ab. Sie wird durch mehrere Effekte gemindert: höhere Abbrecherquoten, höhere Abwanderung nach Studienabschluss, schlechtere Berufschancen (bei mangelnden Sprachkenntnissen) sowie Verbleib ohne
Erwerbstätigkeit. ${ }^{19}$ Die hierzu bekannten Zahlen ${ }^{20}$ sind nicht quantitativ belastbar. Die TU München stellt dennoch derzeit alle Master-Studiengänge auf Englisch um. Kann das unter den gegebenen Umständen als rational gelten oder gar Vorbildcharakter beanspruchen?

Der behauptete Fachkräftemangel sollte sich im Übrigen in einer Marktwirtschaft durch steigende Gehälter zeigen - und auch regulieren. Das ist aber nicht erkennbar. Viele deutsche Akademiker wandern vielmehr ins Ausland ab, nur ein Teil von ihnen kommt zurück. 21

Und das ist nur die wirtschaftliche Seite. Hinzu kommen: die gesellschaftliche Problematik der Integration wegen Abbruchs oder trotz englischsprachigen Abschlusses arbeitsloser Ausländer ohne für eine Erwerbstätigkeit ausreichende Deutschkenntnisse und die Fragen, ob ein solcher provozierter Brain-Drain unethische Politik wäre, weil er den Herkunftsländern schadet.

\section{Schlusswort}

Die Hochschulen sollen nach wie vor ein angemessenes Volumen an englischsprachigen Lehrveranstaltungen für die Gaststudierenden anbieten können. Die einheimischen Studierenden sollen weiterhin die Möglichkeit haben, im Studium die für Auslandssemester, internationale Jobs und wissenschaftliche Kommunikation insgesamt erforderlichen Sprachfähigkeiten zu erwerben, soweit erwünscht und nicht bereits vorhanden. Dieser Spracherwerb muss nicht auf Englisch beschränkt sein. Einzelne fremdsprachliche Lehrveranstaltungen können diese Sprachfähigkeiten unterstützen, soweit Professoren sich dafür entscheiden. Die staatlichen Hochschulen müssen v. a. dem Bildungsauftrag gegenüber der jüngeren Generation und der Gesellschaft folgen. Dieser kann nicht in der Verdrängung der landessprachlichen Hochschullehre bestehen. Es darf dabei auch nicht verkannt werden, dass die deutschen Fachhochschulen in erster Linie für die nationalen und regionalen Arbeitsmärkte ausbilden. Die von vielen Hochschulleitungen - der vermeintlichen Modernität folgend forcierte Abschaffung der Landessprachen im Studium ist hierfür nachteilig. Eher verletzt sie die Freiheitsrechte der Wissenschaftler und beraubt die Studierenden der Chance, sich die Wissenschaftssprache der eigenen Kulturgemeinschaft anzueignen und die sprachvermittelte Wissenschaftstradition zu verstehen. Lieb gewonnene Internationalisierungsnarrative wie englischsprachige Studiengänge seien notwendig, um ausländische Studierende als künftige Fachkräfte zu gewinnen, oder man verdiene einzig durch Lehre in Englisch die Bezeichnung „international“ haben ausgedient. Die kulturellen, bildungspolitischen ethischen und ökonomischen Folgen von Sprachalternativen müssen innerwissenschaftlich analysiert und entschieden werden. 


\section{Literatur}

ADAWIS (Hrsg.) (2013): „Die Sprache von Forschung und Lehre: Welche, Wo, für Wen?”, Verlag Deutsche Sprache Paderborn.

ADAWIS (Hrsg.) (2014): „Die Sprache von Forschung und Lehre: Bindeglied der Wissenschaft zu Kultur und Gesellschaft", Verlag Deutsche Sprache Paderborn.

Bongo, G. (2006): Asymmetrien in wissenschaftlicher Kommunikation, in: Ehlich, K./Heller, D. (Hrsg.) (2006), S. 175-190.

Ehlich, K. (2000: 7 f.): Deutsch als Wissenschaftssprache für das 21. Jahrhundert, http://www.gfl-journal.de/1-2000/ehlich. $\mathrm{html}(18.07 .18)$.

Ehlich, K. (2006): Mehrsprachigkeit in der Wissenschaftskommunikation - Illusion oder Notwendigkeit?, in: Ehlich, K./Heller, D. (Hrsg.) (2006): Die Wissenschaft und ihre Sprachen, Verlag Peter Lang, li52, Bern u. a., S. 17-38.

Mocikat, R./Dieter, H. (2017): Zeichen der Internationalität oder ideologische Nebelkerze?, in DHN 2017/06, S. 30-31.

Oberreuter, H./Krull, W./Meyer, H. J./Ehlich, K. (Hrsg.) (2012): Deutsch in der Wissenschaft. Ein politischer und wissenschaftlicher Diskurs, Verlag Olzog, München.

Petereit, K./Spielmanns-Rome, E. (2010): Sprecht Deutsch mit uns. Ausländische Studierende in englischsprachigen Studiengängen wollen mehr Deutsch lernen, in: Forschung und Lehre 3/10, S. 172-173.

1 Kretzenbacher, H. L.: (1998:134), zitiert nach Bongo, G. (2006:175).

2 Ehlich, K. (2000:7f), Ehlich/Heller (2006).

3 Z. B.: „Internationalisierung an den Hochschulen - Niederlande treten auf die Bremse”, in Deutschlandfunk vom 06.06.2018, Beitrag von Andrea Lueg; od. Kroatien, Universität Zagreb: https://networks.h-net.org/node/79435/discussions/1674132/ cfp-die-anglophonisierung-der-wissenschaftssprache.

4 Z. B. in Deutschland „DAAD Memorandum zur Förderung des Deutschen als Wissenschaftssprache” (2010), oder in den Niederlanden "Manifest zur Wissenschaftssprache" (2015): http://adawis.de/fileadmin/user_upload/Seiten/Aktuelles/ Manifest_Niederlande_2015.pdf u. a.

5 Vgl. http://adawis.de/ausland/italien/(18.07.2018).

6 Vgl. Ehlich, K. (2006:30), ADAWIS (Hrsg.) (2014).

7 https://www.br.de/nachrichten/tu-muenchen-englisch-unsinn-zukunft-100.html (18.07.2018).

8 Z. B. „Ordnung über die Vergabe von Leistungsbezügen und Forschungs- und Lehrzulagen an Professorinnen und Professoren der Technischen Hochschule Wildau" 4 §4, S. 9.

9 Vgl. Ehlich, K. (2006:33 f.), Oberreuter, H./et al. (Hrsg.) (2012); Mocikat, R./Dieter, H. (2017:30-31); ADAWIS (2013, 2014).

10 Z.B. http://adawis.de/aktionen/veranstaltungen-des-adawis/ (18.07.2018).

11 Z.B.: https://www.br.de/nachrichten/oberbayern/inhalt/wie-die-englische-sprache-die-wissenschaft-beherrscht-100. $\mathrm{html}(18.07 .2018)$.

12 Die Ideen der Entwicklung von europäischen „mehrsprachigkeitsoffenen Zitationsindices” (Ehlich, 2006:36 f.) finden sich in verschiedenen Varianten in vielen Publikationen.

13 Die Zahl schwankt zw. 70 Prozent laut der Studie des SVR „Von Hörsaal in den Betrieb?” (2017:5) und 54 Prozent laut "Empfehlungen des Wissenschaftsrates zur Internationalisierung von Hochschulen" (2018:138).

14 Petereit, K./Spielmanns-Rome, E. (2010).

15 http://adawis.de/fileadmin/user_upload/PM_Tutzinger_Erklaerung_2018.pdf (18.07.2018).

16 S. dazu auch „Empfehlungen des Wissenschaftsrates ..." (2018:11) (s.o.).

17 Gutachten „Internationalisierung der Hochschulen“ des Aktionsrates Bildung (2012:53).

18 Bildung auf einen Blick 2015: - OECD-Indikatoren (2015:185-186).

19 Vgl. Empfehlungen des Wissenschaftsrates (2018:140): ebd.

20 Hierzu z. B: Vom Hörsaal in den Betrieb? Studie des SVR-Forschungsbereichs (2017-5, S. 5 ff).

21 Dazu z. B.: International Mobil - Motive, Rahmenbedingungen und Folgen der Aus- und Rückwanderung deutscher Staatsbürger, Studie des SVR-Forschungsbereichs (2015-1). 